\title{
Echocardiographic Features in Bartonella Endocarditis: A Case Series
}

\author{
Orathai Pachirat ${ }^{\mathrm{a}, \mathrm{d}}$, Sompop Prathanee ${ }^{\mathrm{b}}$, George Watt ${ }^{\mathrm{c}}$
}

\begin{abstract}
Bartonella spp. are emerging pathogens that are reported as the cause of blood culture-negative endocarditis ( $\mathrm{BCNE}$ ). However, echocardiographic features and assessment of this endocarditis remains unclear. Four patients with $B$. henselae endocarditis were identified. All patients had underlying cardiac conditions: rheumatic heart disease in three, congenital heart disease in one. Evidence of vegetations was found on the aortic valve in all patients with large, highly mobile vegetations and severely destroyed valves demonstrated by the transthoracic echocardiogram leading to severe aortic regurgitation and heart failure. The vegetations were found on both the aortic and the mitral valve in two patients. All patients had negative blood cultures and underwent urgent valves replacement due to heart failure with good clinical outcome. The diagnosis of $B$. henselae endocarditis is based mainly on clinical suspicion in BCNE, specific serologic testing and polymerase chain reaction (PCR) detection on excised valve tissue.
\end{abstract}

Keywords: Infective endocarditis; Bartonella endocarditis; Echocardiographic features; Blood culture negative endocarditis; PCR; Bartonella henselae

\section{Introduction}

Bartonella endocarditis is a zoonotic disease, serious medical condition, potentially fatal, caused by Bartonella spp. which are facultative intracellular bacteria, small Gram- negative bacilli, fastidious organism that cause BCNE and have been increasingly reported [1]. The serological analysis using indirect immunofluorescence assay (IFA) remains the most common

Manuscript submitted January 23, 2018, accepted February 8, 2018

aDepartment of Medicine, Faculty of Medicine, Khon Kaen University, KhonKaen 40002, Thailand

bDepartment of Surgery, Faculty of Medicine, Khon Kaen University, KhonKaen 40002, Thailand

'International Emerging Infections Program, Thailand MOPH-US CDC Collaboration, Nontaburi, Thailand

${ }^{\mathrm{d} C}$ Corresponding Author: Orathai Pachirat, Cardiovascular Unit, Faculty of Medicine, Khon Kaen University, 123 Mitraparp Highway, KhonKaen 40002, Thailand. Email: orathai@hotmail.com

doi: https://doi.org/10.14740/cr595w methods for diagnosing endocarditis caused by these bacteria. However, these cases of infective endocarditis (IE) can also be successfully diagnosed by polymerase chain reaction (PCR), allowing for the detection of Bartonella DNA in blood or resected heart valves $[2,3]$.

The echocardiography has to be performed in all cases which are suspected of IE. It is an extremely important tool in the diagnosis of IE, combining the advantages of identifying vegetations, abscesses, and new prosthetic valve dehiscence, which are hallmarks of IE, assessing the severity of valve damage, detecting cardiac complications and predicting embolic risk [4]. The transthoracic echocardiography (TTE) must be performed firstly, then the transesophageal echocardiography (TEE) is mandatory in case of doubtful TTE examination, in prosthetic valve IE and when the complication of IE is suspected. Bartonella IE is a rare clinical entity, however, its prevalence has increased over the last decade, most often manifesting as BCNE [5]. However, the echocardiographic features of Bartonella IE are not well known because scanty information is available in the literature. The purpose of this study is to present the echocardiographic features and clinical outcome observed in four patients with Bartonella endocarditis.

\section{Case Reports}

A prospective study of the etiology and characteristics of IE at Srinagarind Hospital and Queen Sirikit Heart Center, Khon Kaen University was performed between January 2010 and December 2012. One hundred and fourteen patients were identified to have a clinical diagnosis of IE using modified Duke criteria [6]. TTE was performed in all patients, using previously reported techniques [7]. The hallmark features of IE are vegetations attach on the valves which can occationally occur at unusual sites where the endocardium has been disrupted by abnormal flow (along regurgitation jets), that were demonstrated by TTE.

Four cases of $B$. henselae IE were diagnosed. Age at echocardiographic diagnosis of IE was 22 - 57 years. Two patients were male. All patients had underlying cardiac conditions: rheumatic heart disease in three, congenital heart disease in one. Evidence of vegetations was found on the aortic valve in all patients with large, highly mobile vegetations and severely destroyed valves demonstrated by TTE, leading to severe aortic regurgitation and heart failure. Evidence of vegetations was found on both the aortic and the mitral valve in two patients. 


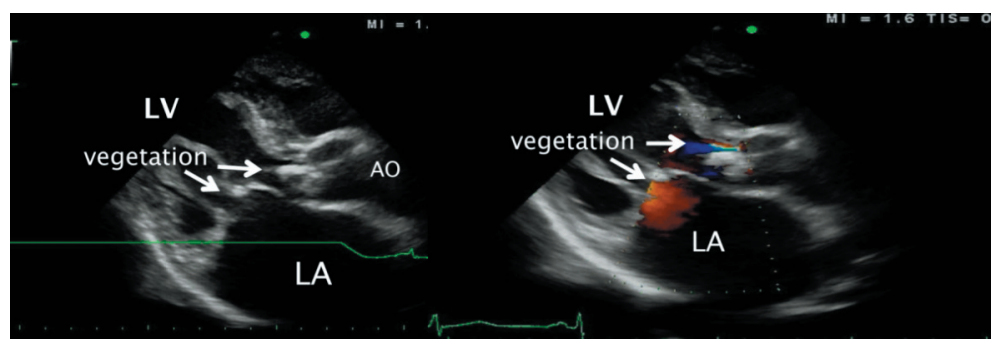

Figure 1. Transthoracic echocardiography revealed large vegetations on the aortic valve and mitral valve with underlying rheumatic heart disease.

All patients had negative blood cultures and underwent urgent valve replacement with good clinical outcome and without inhospital mortality.

\section{Case 1}

A 57-year old male with underlying rheumatic heart disease presented to a local hospital with the history of fever, myalgia and shortness of breath for 10 days. He was transferred to our cardiac center. On admission, he had high fever, heart murmurs consistent with aortic stenosis and regurgitation, mitral stenosis and regurgitation with signs of congestive heart failure. The TTE showed large mobile vegetations on the aortic valve and the mitral valve (Fig. 1). Intravenous ampicillin and gentamicin were begun on admission. However, symptoms of congestive heart failure worsened, the patient underwent urgent aortic and mitral valve replacement. Histopathological examination of the heart valve tissue showed active endocarditis. Three sets of routine blood cultures were negative. Serologic testing was performed using an indirect immunofluorescent antibody assay which demonstrated for $B$. henselae with serum antibody titer of 1:512 on admission. $B$. henselae was also demonstrated in heart valve tissue by PCR, and by immunohistochemical staining. The patient was started on therapy for Bartonella IE with intravenous ampicillin and gentamicin for 6 weeks and was discharged from the hospital with clinical improvement and good outcome.

\section{Case 2}

A 45-year-old male with underlying rheumatic heart disease who was transferred to our center because of shortness of breath, orthopnea for 1 week and 3 months of fever and weight loss. On admission, he was found in severe heart failure, heart murmur consistent with aortic regurgitation, mitral stenosis and regurgitation. The TTE demonstrated mobile vegetations on the aortic valve with severe aortic regurgitation and on the mitral valve (Fig. 2). Intravenous ampicillin and gentamicin were begun on admission. The patient underwent urgent aortic and mitral valve replacement due to intractable heart failure. Three sets of blood culture were negative but $B$. henselae was demonstrated in the excised heart valve tissue by serology, PCR and immunohistochemical staining. The patient's overall clinical condition improved soon after surgery, and he was discharged from the hospital within 3 weeks. The patient remained clinical stable at 1-year follow-up.

\section{Case 3}

A 48-year-old female with underlying rheumatic heart disease, presented with low grade fever for 1 month. One week before she came to hospital, patient developed dyspnea on exertion, orthopnea and ankle edema of both legs. On admission, her vital sign was stable. She was found to be in congestive heart failure. She had the heart murmur consistent with aortic regurgitation, aortic stenosis and mitral stenosis. The TTE demonstrated mobile vegetations on the aortic valve and destroyed aortic cusp with severe aortic regurgitation, moderate aortic stenosis and moderate mitral stenosis (Fig. 3). Intravenous ceftriaxone and gentamicin were begun with clinically improvement but the patient developed heart failure again due to severe aortic regurgitation. The patient underwent urgent aortic and mitral valve replacement. Three sets of blood culture were negative. $B$. henselae was demonstrated in the excised heart valve tissue by serology, PCR and immunohistochemical

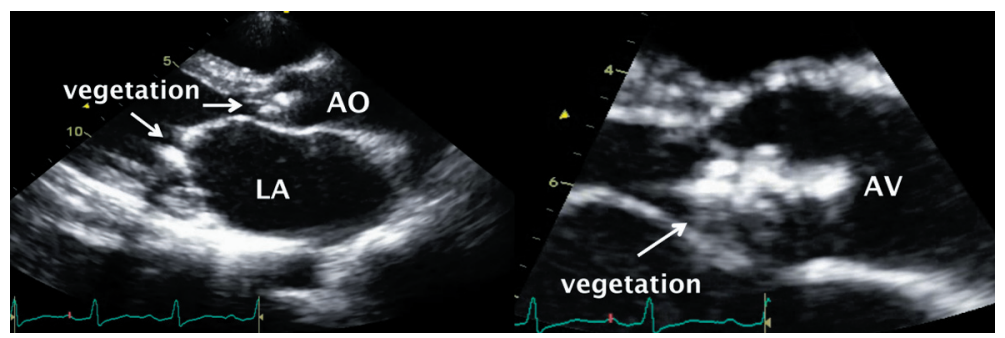

Figure 2. Transthoracic echocardiography revealed vegetations on the aortic valve and mitral valve with perforation of right coronary cusp. 


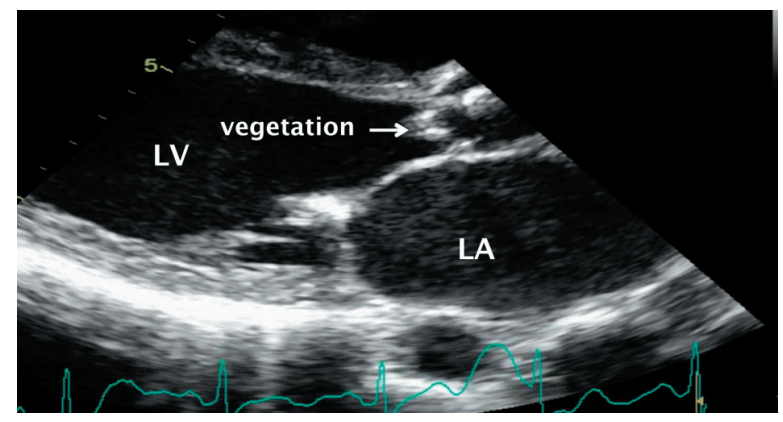

Figure 3. Transthoracic echocardiography revealed vegetation destroyed aortic cusp with underlying rheumatic heart disease.

staining. She was in the hospital for 4 weeks, and discharged from the hospital with good clinical outcome.

\section{Case 4}

A 22-year-old female with underlying congenital heart disease presented with history of fever for 1 week, no night sweat, fatigue and dyspnea on exertion. On admission, her vital sign was stable, mild heart failure, heart murmur consistent with ventricular septal defect (VSD) and severe aortic regurgitation. The TTE demonstrated vegetation at aortic valve with flail right coronary cusp (RCC) which caused severe aortic regurgitation. Subarterial type VSD with herniated RCC was demonstrated with left to right shunt (Fig. 4). Intravenous ampicillin and gentamicin were begun. However, symptoms of congestive heart failure worsened. The patient underwent urgent aortic valve replacement and VSD closure. Three sets of blood culture were negative. $B$. henselae was demonstrated in the excised heart valve tissue by serology, PCR and immunohistochemical staining. She was in the hospital for 6 weeks for antibiotics treatment and discharged from the hospital with good clinical outcome.

\section{Discussion}

Bartonella has remained an uncommon cause of IE. It is a zoonosis associated with significant severe valvular damage in patient who had underlying heart valve defect, often causing congestive heart failure. Our data confirm the occurrence of culture-negative endocarditis due to Bartonella in Thailand. The identification of the causative pathogen is often difficult as a result of negative blood culture, application of molecular technique and serologic testing are mandatory for identification. The echocardiography has become a mainstay in the diagnosis of IE, TTE alone is often sufficient to establish the diagnosis especially in native valve IE. Overall the detection rate of vegetations by TTE in patients with a clinical suspicion of native valve IE averages around 50-60\% [8]. Vegetations on the prosthetic valves are more difficult to detect by TTE than those involving native valves, therefore TEE should always be used if the diagnosis of prosthetic valve IE is suspected [9-11]. In this present study, the diagnosis in all patients was accomplished by TTE examination. The regurgitation of the infected valve is almost constant and results from a variety of mechanisms. Valvular perforation is a frequent complication that may cause severe insufficiency with an acute onset and precipitate heart failure that demonstrated by TTE. In aortic IE, cusp perforation, flail or both may occur in up to $50 \%$ of cases. Severe aortic regurgitation as estimated by Doppler echocardiography has been associated with heart failure and poor prognosis. Perforation of the mitral leaflets is less common, occurring in only $15 \%$ of patients with mitral valve IE especially in rheumatic heart disease. TEE should also be performed to provide a more detailed assessment in the setting of perivalvular extension of infection, but TEE was not performed in our study due to good image quality of the TTE [12].

\section{Conclusions}

In the setting of $B$. henselae IE, the echocardiographic features demonstrate large mobile vegetations and destructive, extensive valvular damage in patients with preexisting heart conditions. The diagnosis is based mainly on clinical suspicion in BCNE, specific serologic testing and PCR detection on excised valve tissue. Early surgical intervention is associated with better long-term outcome.

\section{Acknowledgments}

This work was supported by the Global Disease Detection Program and the Division of Vector Borne Infectious Diseases at

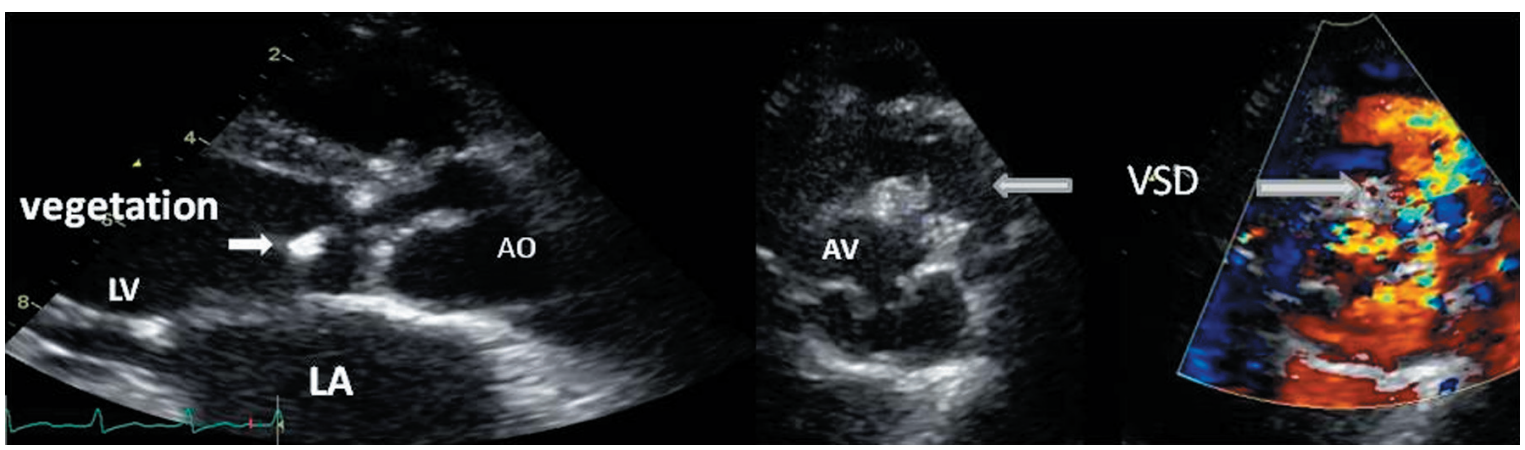

Figure 4. Transthoracic echocardiography showed vegetation on the aortic valve with VSD. 
Centers for Disease Control and Prevention, USA and the University of the Mediterranean, Marseille, France.

\section{Conflict of Interest}

The authors declare no conflict of interest.

\section{References}

1. Edouard S, Nabet C, Lepidi H, Fournier PE, Raoult D. Bartonella, a common cause of endocarditis: a report on 106 cases and review. J Clin Microbiol. 2015;53(3):824829.

2. Fournier PE, Thuny F, Richet H, Lepidi H, Casalta JP, Arzouni JP, Maurin M, et al. Comprehensive diagnostic strategy for blood culture-negative endocarditis: a prospective study of 819 new cases. Clin Infect Dis. 2010;51(2):131-140.

3. Lamas Cda C, Ramos RG, Lopes GQ, Santos MS, Golebiovski WF, Weksler C, Ferraiuoli GI, et al. Bartonella and Coxiella infective endocarditis in Brazil: molecular evidence from excised valves from a cardiac surgery referral center in Rio de Janeiro, Brazil, 1998 to 2009. Int J Infect Dis. 2013;17(1):e65-66.

4. Evangelista A, Gonzalez-Alujas MT. Echocardiography in infective endocarditis. Heart. 2004;90(6):614-617.

5. Dreier J, Vollmer T, Freytag CC, Baumer D, Korfer R, Kleesiek K. Culture-negative infectious endocarditis caused by Bartonella spp.: 2 case reports and a review of the literature. Diagn Microbiol Infect Dis.
2008;61(4):476-483.

6. Li JS, Sexton DJ, Mick N, Nettles R, Fowler VG, Jr., Ryan T, Bashore T, et al. Proposed modifications to the Duke criteria for the diagnosis of infective endocarditis. Clin Infect Dis. 2000;30(4):633-638.

7. Tajik AJ, Seward JB, Hagler DJ, Mair DD, Lie JT. Twodimensional real-time ultrasonic imaging of the heart and great vessels. Technique, image orientation, structure identification, and validation. Mayo Clin Proc. 1978;53(5):271-303.

8. Reynolds HR, Jagen MA, Tunick PA, Kronzon I. Sensitivity of transthoracic versus transesophageal echocardiography for the detection of native valve vegetations in the modern era. J Am Soc Echocardiogr. 2003;16(1):6770 .

9. Erbel R, Rohmann S, Drexler M, Mohr-Kahaly S, Gerharz $\mathrm{CD}$, Iversen $\mathrm{S}$, Oelert $\mathrm{H}$, et al. Improved diagnostic value of echocardiography in patients with infective endocarditis by transoesophageal approach. A prospective study. Eur Heart J. 1988;9(1):43-53.

10. Shively BK, Gurule FT, Roldan CA, Leggett JH, Schiller NB. Diagnostic value of transesophageal compared with transthoracic echocardiography in infective endocarditis. J Am Coll Cardiol. 1991;18(2):391-397.

11. San Roman JA, Vilacosta I, Zamorano JL, Almeria C, Sanchez-Harguindey L. Transesophageal echocardiography in right-sided endocarditis. J Am Coll Cardiol. 1993;21(5):1226-1230.

12. Shapiro SM, Young E, De Guzman S, Ward J, Chiu CY, Ginzton LE, Bayer AS. Transesophageal echocardiography in diagnosis of infective endocarditis. Chest. 1994;105(2):377-382. 